\title{
ARTÍCULOS
}

\section{ALTMÉTRICAS A NIVEL INSTITUCIONAL: VISIBILIDAD EN LA WEB DE LA PRODUCCIÓN CIENTÍFICA DE LAS UNIVERSIDADES ESPAÑOLAS A PARTIR DE ALTMETRIC.COM}

\author{
Altmetrics at institutional level: Visibility on the web of \\ the scientific production of Spanish universities from \\ Altmetric.com
}

\section{Daniel Torres-Salinas, Pedro-Ángel Castillo-Valdivieso, Álvaro Pérez-Luque y Esteban Romero-Frías}

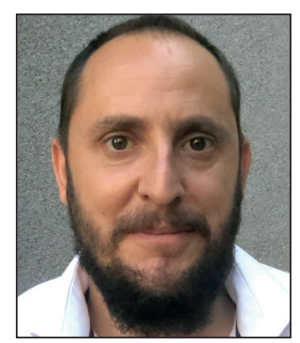

Daniel Torres-Salinas es responsable de la Unidad de Evaluación de la Actividad Científica de la Universidad de Granada (UGR). Es investigador en el grupo de investigación Evaluación de la Ciencia y la Comunicación Científica (EC3) sobre temas bibliométricos. Es CEO de la spin-off de asesoría científica EC3metrics y responsable de la línea de ciencia digital del Medialab UGR. Es co-creador de programas de evaluación de la investigación como CientíficaCVN, Rankings I-UGR de Universidades, Clasificación Integrada de Revistas científicas (CIRC), Bipublishers o UGRinvestiga. Tiene indexadas más de 60 publicaciones científicas en la Web of Science.

http://orcid.org/0000-0001-8790-3314

Universidad de Granada, EC3metrics y Medialab UGR Gran Vía, 48. 18010 Granada, España torressalinas@gmail.com

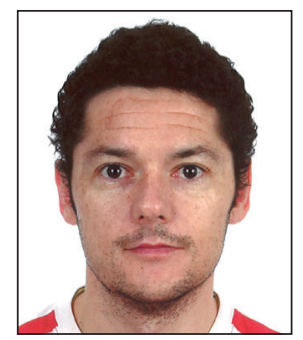

Pedro-Ángel Castillo-Valdivieso es doctor en Informática por la Universidad de Granada (UGR) (2000), donde también obtuvo la licenciatura en Informática (1997) y donde es profesor en el Departamento de Arquitectura y Tecnología de Computadores. Ha sido investigador visitante en Napier University, Edimburgo, y en Santa Fe Institute, Santa Fe, NM, EUA. Ha dirigido proyectos de investigación y co-dirigido cuatro tesis doctorales. Sus áreas de investigación principales son sistemas bio-inspirados, optimización de redes neuronales, predicción de series temporales e inteligencia computacional en juegos.

http://orcid.org/0000-0002-5258-0620

Universidad de Granada Departamento de Arquitectura y Tecnología de Computadores Periodista Daniel Saucedo Aranda. 18071 Granada, España pacv@ugr.es

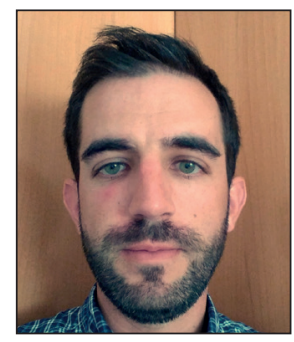

Álvaro Pérez-Luque es graduado en Ingeniería Informática por la Universidad de Granada (UGR), especialidad Tecnologías de la Información, y técnico superior en Administración de Sistemas en Red, por el C.E.S Ramón y Cajal de Granada. Trabaja en el Csirc de la UGR como desarrollador en Oracle Developer. Sus áreas de trabajo son desarrollo y mantenimiento de aplicaciones en Oracle y de aplicaciones basadas en Web Services.

http://orcid.org/0000-0002-5166-5219

Universidad de Granada, Servicio Central de Informática Profesor Adolfo Rancaño. 18071 Granada, España alvaropl@ugr.es 


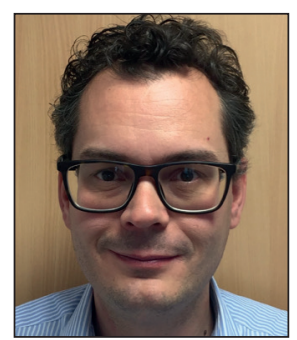

Esteban Romero-Frías es profesor titular de la Universidad de Granada en el Departamento de Economía Financiera y Contabilidad. Actualmente es director de Medialab UGR - Laboratorio de Investigación en Cultura y Sociedad Digital de la Universidad de Granada. Sus trabajos de investigación se han centrado principalmente en la aplicación de técnicas webmétricas al campo de la empresa y la política, así como en el empleo de tecnologías digitales en educación. Trabaja en temas de cultura digital, digital scholarship y ciencias sociales y humanidades digitales.

http://estebanromero.com

http://orcid.org/0000-0003-2205-3560

Universidad de Granada, Medialab UGR, CTT Gran Vía, 48. 18010 Granada, España erf@ugr.es

\section{Resumen}

Este estudio se centra en las publicaciones de las universidades españolas en la Web of Science (WoS) durante 2014-2016 (125.824 artículos) y calcula 17 indicadores con Altmetric.com. Se han establecido tres objetivos: 1) ofrecer una perspectiva general de las altmétricas en las universidades españolas, 2) determinar la cobertura de Altmetric.com en el caso español y 3 ) establecer cuáles son las altmétricas más relevantes. Los resultados evidencian que el $42 \%$ de los artículos en la WoS están indexados en Altmetric.com. Los indicadores generados por Twitter, prensa digital, Facebook y Wikipedia explican el $82 \%$ de la varianza. Como conclusiones se señalan que: a) publicar en revistas de alto impacto y en áreas como Medicina y Biología contribuye a que las universidades obtengan mejores altmétricas; y b) un reducido número de indicadores es suficiente para determinar el impacto altmétrico.

\section{Palabras clave}

Altmétricas; Universidades; España; Altmetric.com; Indicadores bibliométricos; Producción científica; Visibilidad; Redes sociales; Twitter; Facebook; Wikipedia.

\section{Abstract}

This study focuses on the publications of Spanish universities indexed in the Web of Science (WoS) during 2014-2016 $(125,824$ articles) and calculates 17 indicators with Altmetric.com. Three objectives have been established: 1) to offer a general perspective of the altmetrics in Spanish universities, 2) to determine the coverage of Altmetric.com in the Spanish case and 3) to establish which are the most relevant altmetrics. The results show that $42 \%$ of the articles in the WoS are indexed in Altmetric.com. The indicators generated by Twitter, the digital press, Facebook and Wikipedia explain $82 \%$ of the variance. As conclusions, it is pointed out that: a) publishing in high impact journals and in areas such as Medicine and Biology helps universities to obtain better altmetrics; and b) a small number of indicators is enough to determine the altmetric impact.

\section{Keywords}

Altmetrics; Spanish universities; Altmetric.com; Bibliometrics indicators; Scientific production; Visibility; Social networks; Twitter; Facebook; Wikipedia.

Torres-Salinas, Daniel; Castillo-Valdivieso, Pedro-Ángel; Pérez-Luque, Álvaro; Romero-Frías, Esteban (2018). "Altmétricas a nivel institucional: visibilidad en la Web de la producción científica de las universidades españolas a partir de Altmetric.com". El profesional de la información, v. 27, n. 3, pp. 483-492.

https://doi.org/10.3145/epi.2018.may.03

\section{Introducción}

La popularización de la Web en la última década, particularmente la denominada web 2.0, ha permitido conocer mejor los impactos de la producción científica, tanto en el propio entorno académico, como en los medios de comunicación de masas, y los medios sociales (Piwowar, 2013a). La expansión de las redes sociales ha tenido un doble efecto:

- los productos académicos tradicionales (artículos, libros, etc.) se comunican a través de múltiples vías tanto a audiencias académicas como no académicas;

- la variedad de productos resultantes del trabajo investigador se ha incrementado considerablemente, convirtiéndose los académicos en los propios gestores de sus identidades digitales (Weller, 2011) y en responsables de la difusión de su investigación a través de publicaciones en abierto, perfiles en redes, etc.
En los años 90 del siglo pasado apareció la webmetría (TheIwall; Vaughan; Björneborn, 2005), centrada en el estudio cuantitativo de la Web aplicando conceptos y técnicas propias de la bibliometría. A pesar de que la webmetría amplió la mirada hacia otras áreas de conocimiento como la empresa (Vaughan; Wu, 2004) o la política (Romero-Frías; Vaughan, 2012), Thelwall (2008) subrayaba cómo su objeto de interés central eran los problemas propios de la bibliometría. En una línea similar Bar-Ilan (2008) definía el objeto de la webmetría en su relación con cuestiones como los índices de impacto web, sitios web de revistas y de universidades, etc. Si a este escenario añadimos el citado auge de las redes sociales y su empleo con fines académicos, nos encontramos ante la aparición de la altmetría (Piwowar, 2013a, 2013b) o de lo que en su momento se denominó scientometrics 2.0 (Priem; Hemminger, 2010). Para Priem et al. (2010) las altmétricas constituyen una rama de estudio dedicada a 
la creación y análisis de indicadores basados en redes digitales para la evaluación de la actividad académica. Se trata pues de una respuesta cuantitativa para medir la visibilidad de la ciencia en las plataformas donde ésta se comunica.

Las expectativas generadas en torno a los indicadores altmétricos han sido grandes, ya sea como potenciales sustitutos de los sistemas basados en citas, ya sea como complemento de éstos (Bornmann, 2014; Costas; Zahedi; Wouters, 2015; Haustein, 2016; Wilsdon et al., 2015). Wouters y Costas (2012) identifican cuatro argumentos a favor de las altmétricas:

- posibilidad de analizar diversos canales de diseminación de la información, no sólo libros y revistas;

- velocidad con la que se pueden tener datos;

- transparencia de los métodos empleados;

- posibilidad de medir el impacto más allá del ámbito académico.

Estos argumentos, al tiempo que apuntan el potencial de estos métodos, nos enfrentan a otros tantos problemas y desafíos. Son varios los retos relacionados con los indicadores altmétricos.

Uno de los más importantes es cuál es su significado preciso y qué tipo de impacto refleja: ¿social o científico? (Sud; TheIwall, 2014; Haustein, Bowman; Costas, 2015).

Otro es cómo abordar la medición de un conjunto de indicadores que escapan a una formalización tan consolidada como la que nos ofrecen los indicadores bibliométricos.

Torres-Salinas, Cabezas-Clavijo y Jiménez-Contreras (2013) recuerdan que una de las limitaciones de las altmétricas es la escasa validez de algunos de esos indicadores para representar la actividad científica.

También cabe reseñar la inconsistencia en la recuperación de los datos a través de códigos identificativos (Robinson-García et al., 2014) y la desigual cobertura que proporcionan las diversas fuentes de información (Haustein, 2016; Gumpenberger; Glänzel; Gorraiz, 2016).

Los principales servicios que proporcionan información altmétrica de forma masiva son comerciales, encontrándose entre los más utilizados y estudiados Altmetric.com (Robinson-García et al., 2014) y PlumX (Torres-Salinas; Gumpenberger; Gorraiz, 2017).

Altmetric.com es el servicio de referencia en esta materia por la variedad de fuentes que proporciona y por desarrollar el indicador Altmetric attention score, que sintetiza el impacto de las publicaciones digitales en diversos medios y redes. Se trata sin embargo de un indicador no exento de críticas ya que sintetiza en una puntuación medidas de plataformas muy dispares con una ponderación poco justificada (Gumpenberger; Glänzel; Gorraiz, 2016) que le da un fuerte carácter subjetivo (Liu; Adie, 2013). Asimismo un indicador como el número de lecturas de Mendeley se excluye del cálculo del Altmetric attention score ya que no se puede verificar (Altmetric.com, 2017a), por lo que los promotores del Manifiesto de Leiden (Hicks et al., 2015) recomiendan prescindir de las mismas.

Al margen de estas limitaciones, gracias a este tipo de apli- caciones los trabajos sobre altmétricas han proliferado en los últimos años: sólo en Web of Science hay 244, de ellos 15 revisiones. A nivel general los trabajos más frecuentes en este campo exploran:

- relaciones entre los indicadores altmétricos y bibliométricos (Thelwall et al., 2013);

- utilidad de las nuevas métricas para analizar otros tipos documentales (Peters et al., 2016);

- obertura y fiabilidad de las fuentes (Torres-Salinas; Robinson-García; Gorraiz, 2017).

A nivel agregado se han estudiado sobre todo disciplinas (Mohammadi; Thelwall, 2014) y la figura del investigador (Haustein et al., 2014a; Torres-Salinas; Milanés-Guisado, 2014; Martín-Martín; Orduña-Malea; Delgado-López-Cózar, 2016). Sin embargo no se ha prestado atención a la aplicación de las altmétricas a nivel institucional. Torres-Salinas, Robinson-García y Jiménez-Contreras (2016) realizaron una primera aproximación con el análisis de cuatro universidades españolas con el fin de testear una metodología viable y valorar la aplicación de los indicadores altmétricos a instituciones.

Nuestro objetivo general es determinar la visibilidad de la producción científica de las universidades españolas en las plataformas que rastrea Altmetric.com con el objeto de valorar la viabilidad y limitaciones de este enfoque como posible instrumento de evaluación científica. Más concretamente los objetivos específicos son:

- Ofrecer una perspectiva general de la visibilidad de la investigación producida por las universidades españolas en las plataformas digitales recogidas en Altmetric.com.

- Analizar el grado de cobertura de los trabajos de Web of Science recogidos en Altmetric.com con el fin de comprobar su alcance y validez como fuente de información para el análisis de la producción científica de las universidades españolas.

- Determinar cuáles son los indicadores altmétricos más relevantes que determinan el impacto en medios sociales de la investigación de las universidades españolas.

\section{Material y métodos}

El trabajo se centra en analizar el impacto de los resultados científicos en medios/plataformas digitales, para lo cual hemos considerado como investigación los resultados publicados por universidades españolas en revistas científicas indexadas en Web of Science (WoS). Para calcular dicho impacto se ha utilizado como fuente de información la web Altmetric.com (Robinson-García et al., 2014), que captura de forma sistemática información sobre las menciones que recibe un documento en distintas plataformas web (tabla 1).

La muestra de instituciones analizadas se compone de 66 universidades españolas, públicas y privadas. Los pasos realizados para recopilar la información fueron:

- Descarga de los artículos de las universidades indexados en WoS para el período 2014-2016. El total de artículos analizados ha sido de 125.824 (47.175, publicados en $2014,47.379$ en 2015 y 31.270 en 2016 ). Las consultas se realizaron en octubre de 2016.

- Identificación de los artículos WoS con DOI y búsque- 
Tabla 1. Plataformas e indicadores altmétricos disponibles en Altmetric.com

\begin{tabular}{|l|l|}
\hline N. de usos en Mendeley (readers) & N. de menciones en publicaciones F1000 \\
\hline N. de menciones en Twitter & N. de menciones en publicaciones de Weibo \\
\hline N. de menciones en noticias de prensa (news stories) & N. de informes técnicos (policy documents) \\
\hline N. de menciones en publicaciones de Facebook & N. de menciones en vídeos (YouTube) \\
\hline N. de menciones en artículos de blog & N. de menciones en publicaciones Q\&A \\
\hline N. de menciones en publicaciones de Google+ & N. de menciones en publicaciones de Linkedln \\
\hline N. de menciones en páginas de Wikipedia & N. de pins (Pinterest) \\
\hline N. de menciones en Reddit & N. de inclusiones en Syllabi (Open Syllabus) \\
\hline N. de menciones en portales de expertos (peer reviews) & \\
\hline
\end{tabular}

da-descarga de las métricas de éstos a través del buscador Altmetric Explorer. El 92\% de los artículos WoS contaban con DOI y pudieron ser buscados recuperándose indicadores altmétricos para un total de 53.347 artículos. Hay que indicar que Altmetric.com hace un seguimiento de todos los trabajos con DOI, es decir todos aquellos presentes en el Digital Object Identifier System.

- Generación de una base de datos que incluye los artículos WoS vinculados a sus altmétricas y las universidades que los han publicado.

Las principales limitaciones metodológicas se refieren a dos aspectos de Altmetric.com: las fuentes y la cobertura de los DOls (Robinson-García et al., 2014). En relación con las fuentes hay que indicar que apenas se utilizan recursos españoles y que además existe una fuerte concentración de las métricas en determinadas fuentes (Priem; Piwowar; Hemminger, 2012). Por ello para identificar las fuentes de la tabla 1 más relevantes para las universidades españolas se realiza un análisis de componentes principales (PCA). también incorporar herramientas que permitan al lector profundizar en los mismos (top papers, clasificación de las universidades según plataforma, visualizaciones, etc.).

http://altmetrics.knowmetrics.org

Este portal se enmarca en el proyecto de I+D Knowmetrics: evaluación del conocimiento en la sociedad digital. Dado el acceso público al $R K$, el objetivo no es describir en este trabajo la plataforma ni sus funciones sino realizar un análisis de sus datos que nos permita darle un uso útil y contextualizado.

En relación con los indicadores se han estudiado 17 métricas alternativas (tabla 1). Para analizar la importancia de los indicadores se ha determinado el porcentaje de trabajos que reciben al menos una mención para cada plataforma y la Tasa de cobertura de Altmetric.com (en adelante, TCA), finalizando con un análisis de componentes principales para determinar los indicadores que condicionan la visibilidad de las universidades.
Otro problema de Altmetric. com es la utilización del DOI y otros identificadores (Arxiv id, PMID, etc.) como medio de recuperación, perdiéndose las menciones cuando no se citan a través de DOls o URL (Haustein et al., 2014b). La distribución de los DOIs no es ni geográfica ni disciplinarmente homogénea, por lo que determinados perfiles de publicación pueden verse perjudicados. Este aspecto se analiza a través del indicador Tasa de cobertura en Altmetric.com (TCA) que para el conjunto de las universidades se sitúa en el $42 \%$.

El conjunto de datos empleados se ha hecho público con el fin de permitir la reproducibilidad a través del Ranking Knowmetrics (RK; imagen 1$)$, con el cual no sólo se pretende liberar los datos, sino

\begin{tabular}{|c|c|c|c|c|c|}
\hline $\begin{array}{l}\text { Universidad } I \\
\text { Knowics }\end{array}$ & $\begin{array}{c}\text { Número } \\
\text { WOS } \\
end{array}$ & $\begin{array}{l}\text { Número } \\
\text { WOS } \\
\text { Altmetric } \\
end{array}$ & $\begin{array}{l}\text { wos } \\
\text { Altmetric/ } \\
\text { wos } \downarrow\end{array}$ & $\begin{array}{c}\text { Total } \\
\text { Altmetric } \\
\text { Score } \\
end{array}$ & $\begin{array}{c}\text { Total } \\
\text { Menciones en } \\
\text { Altmetric } \mathbb{}\end{array}$ \\
\hline Universitat de Barcelona & 16482 & 9866 & $60 \%$ & 117208 & 323073 \\
\hline $\begin{array}{l}\text { Universitat Autònoma de } \\
\text { Barcelona }\end{array}$ & 10077 & 5682 & $56 \%$ & 55271 & 160592 \\
\hline $\begin{array}{l}\text { Universidad Complutense } \\
\text { de Madrid }\end{array}$ & 7923 & 3339 & $42 \%$ & 36078 & 85772 \\
\hline Universitat de València & 7208 & 3441 & $48 \%$ & 42530 & 97281 \\
\hline
\end{tabular}

\section{Consulta y acceso a los artículos con mayor Altmetric Score por universidad}

\begin{tabular}{|c|c|c|c|c|}
\hline \multicolumn{5}{|l|}{ Altmetric } \\
\hline Score & Doi & & Título & Revista \\
\hline 3023 & 10.1126/science.aac 4716 & Ver & $\begin{array}{l}\text { Estimating the reproducibility of psychological } \\
\text { science }\end{array}$ & SCIENCE \\
\hline 1057 & $10.1038 /$ nclimate 2554 & Ver & $\begin{array}{l}\text { Exceptional twentieth-century slowdown in Atlantic } \\
\text { Ocean overturning circulation }\end{array}$ & $\begin{array}{l}\text { NATURE CLIMATE } \\
\text { CHANGE }\end{array}$ \\
\hline 1051 & $10.1038 /$ nature 12788 & Ver & $\begin{array}{l}\text { A mitochondrial genome sequence of a hominin } \\
\text { from Sima de los Huesos }\end{array}$ & NATURE \\
\hline
\end{tabular}

Imagen 1. Algunas de las funciones del portal Knowmetrics: el ranking general y el acceso a los trabajos más relevantes para cada universidad 
Tabla 2. Indicadores altmétricos de la producción científica en Web of Science de las universidades españolas en su conjunto calculados a partir de Altmetric.com para el período 2014-2016

\begin{tabular}{|c|c|c|c|c|}
\hline & $\begin{array}{c}\text { Total } \\
\text { agregado }\end{array}$ & $\begin{array}{l}\% \text { trabajos } \\
\text { con al menos } \\
1 \text { mención }\end{array}$ & $\begin{array}{l}\text { Promedio } \\
\text { por } \\
\text { universidad }\end{array}$ & $\begin{array}{l}\text { Promedio y des- } \\
\text { viación estándar } \\
\text { por trabajo }\end{array}$ \\
\hline $\begin{array}{l}\text { Total menciones } \\
\text { incluyendo Mendeley }\end{array}$ & 1.439 .521 & 99 & 31.185 & $27,45(s d-65)$ \\
\hline $\begin{array}{l}\text { Total menciones } \\
\text { sin incluir Mendeley }\end{array}$ & 421.786 & 93 & 10.140 & $7,8(s d-35,59)$ \\
\hline N. de usos en Mendeley (readers) & 1.017 .735 & 91 & 21.057 & $19,08(s d-34,5)$ \\
\hline N. de menciones en Twitter & 346.378 & 13 & 8.332 & $6,49(s d-35,08)$ \\
\hline N. de menciones en noticias de prensa (news stories) & 28.427 & 10 & 694 & $0,53(s d-4,31)$ \\
\hline N. de menciones en publicaciones de Facebook & 27.708 & 21 & 656 & $0,52(s d-3,39)$ \\
\hline N. de menciones en artículos de blogs & 7.908 & 7 & 191 & $0,15(s d-1,23)$ \\
\hline N. de menciones en artículos de Google+ & 5.549 & 4 & 129 & $0,10(s d-1,73)$ \\
\hline N. de menciones en páginas de Wikipedia & 1.944 & 2 & 48 & $0,04(s d-0,78)$ \\
\hline N. de menciones en Reddit & 1.108 & 1 & 26 & $0,02(s d-0,24)$ \\
\hline N. de menciones en portales de expertos (peer reviews) & 853 & 1 & 24 & $0,02(s d-0,96)$ \\
\hline N. de menciones en publicaciones F1000 & 616 & 1 & 13 & $0,01(s d-0,12)$ \\
\hline N. de menciones en publicaciones de Weibo & 593 & $<1$ & 13 & $0,01(s d-0,61)$ \\
\hline N. de menciones en informes técnicos (policy documents) & 306 & $<1$ & 6 & $0,01(s d-0,1)$ \\
\hline N. de menciones en vídeos (YouTube) & 304 & $<1$ & 7 & $0,01(s d-0,14)$ \\
\hline N. de menciones en publicaciones de $Q \& A$ & 92 & $<1$ & 2 & $0(s d-0,06)$ \\
\hline N. de menciones en publicaciones Linkedln & 0 & 0 & 0 & $0(s d-0)$ \\
\hline N. de pins en Pinterest & 0 & 0 & 0 & $0(s d-0)$ \\
\hline N. de inclusiones en Syllabi (Open Syllabus) & 0 & 0 & 0 & $0(s d-0)$ \\
\hline
\end{tabular}

\section{Resultados}

\subsection{Indicadores generales del sistema universitario}

De acuerdo con la tabla 2, los 125.824 artículos publicados por las universidades españolas durante el período 20142016 han recibido 1.439 .521 menciones. En esta cifra total se ha tenido en cuenta la plataforma Mendeley, dando como resultado que el $99 \%$ de los trabajos recibe algún tipo de métrica. Si excluimos Mendeley, debido a las limitaciones expresadas anteriormente, el total de menciones se reduce a 421.786 , con un promedio global de 7,8 por trabajo. Los indicadores con más menciones son: Twitter (346.378), plataformas de noticias (28.427) y Facebook (27.708), acumulando conjuntamente el $94 \%$ de las menciones recopiladas de Altmetric.com. Son además las únicas tres plataformas en las que al menos el $10 \%$ de los artículos publicados reciben alguna mención.

Es reseñable que el resto de plataformas apenas generan

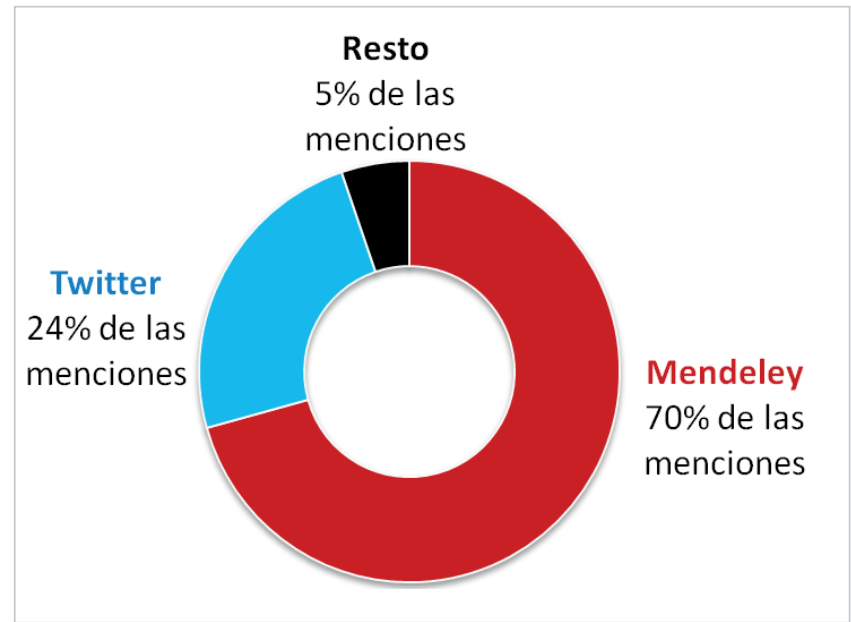

a) Porcentaje considerando el dato relativo a Mendeley

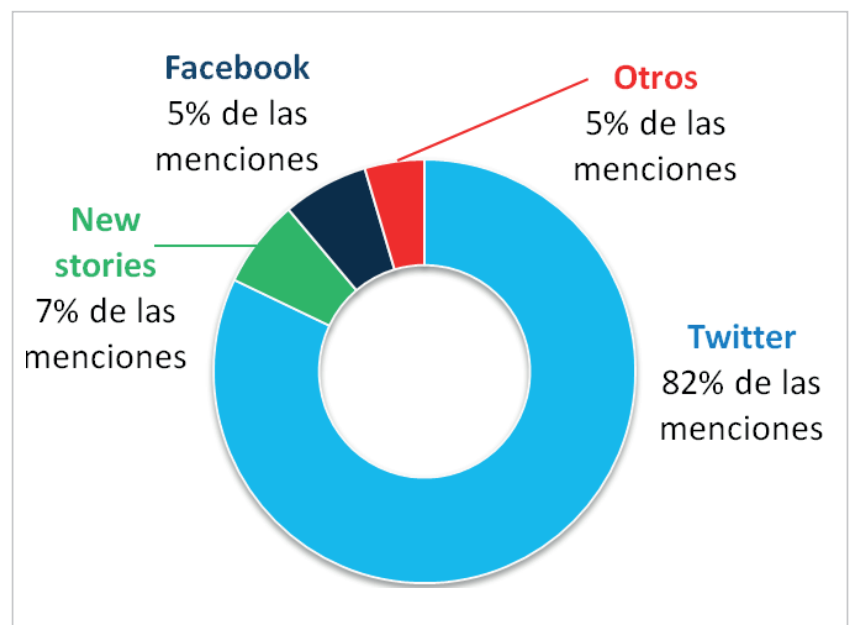

b) Porcentaje sin considerar el dato relativo a Mendeley

Gráfico 1. Porcentaje que representa cada plataforma sobre el total de menciones recopiladas por Altmetric.com para la producción científica Web of Science de las universidades españolas durante 2014-2016 
métricas para las universidades españolas: para 11 de las plataformas analizadas sólo un $2 \%$ de los trabajos, o menos en algunas, reciben como mínimo una mención; asimismo tres de las plataformas no presentan métricas (LinkedIn, Pinterest y Open Syllabus). Esta situación hace que existan enormes diferencias en los promedios de menciones. A modo ilustrativo, el promedio en Twitter por universidad se sitúa en 8.332 mientras que las menciones de Wikipedia se reducen a 48 . Todas las plataformas, salvo Mendeley y Twitter, presentan promedios de menciones por trabajo inferiores a 1. El gráfico 1 muestra el porcentaje de menciones que representa cada plataforma sobre el total de menciones recopiladas desde Altmetric.com. Se puede observar como Mendeley es el gran generador de menciones, con un $70 \%$ sobre el resto de redes. Si excluimos esta plataforma, Twitter, con el $82 \%$ se convierte en la gran red de referencia.

\subsection{Cobertura de Altmetric.com de la producción científica WoS}

Una cuestión importante es establecer el grado de cobertura que tiene Altmetric.com de la producción en Web of Science. Para el conjunto de las universidades la Tasa de cobertura en Altmetric.com (TCA) se sitúa en el $42 \%$. Encontramos que 20 universidades tienen entre el 31\%-40\% de su producción indexada en Altmetric.com y 27 universidades entre el 41\%-50\% (gráfico 2). La universidad que más destaca es la Pompeu Fabra cuya TCA alcanza el 71\%. Entre las universidades con mayor producción científica en Web of Science (tabla 3) la TCA oscila entre el 39\% (Sevilla y Zaragoza) y el $60 \%$ (Barcelona).

Las universidades generalistas e históricas presentan tasas similares (Granada, Complutense de Madrid y del País Vasco, por ejemplo, están en el $42 \%$ ). Tienen valoren inferiores a la media universidades que concentran gran parte de su producción científica en ciencias sociales (Carlos III, 25\%) e ingenierías (Politécnica de Madrid, 26\%; Politècnica de Catalunya, 34\%; Vigo, 33\%). Las instituciones mejor representadas son las que tienen su producción científica orientada a las ciencias de la salud y biológicas (por ejemplo, la Universitat Pompeu Fabra concentra el $75 \%$ de sus publicaciones

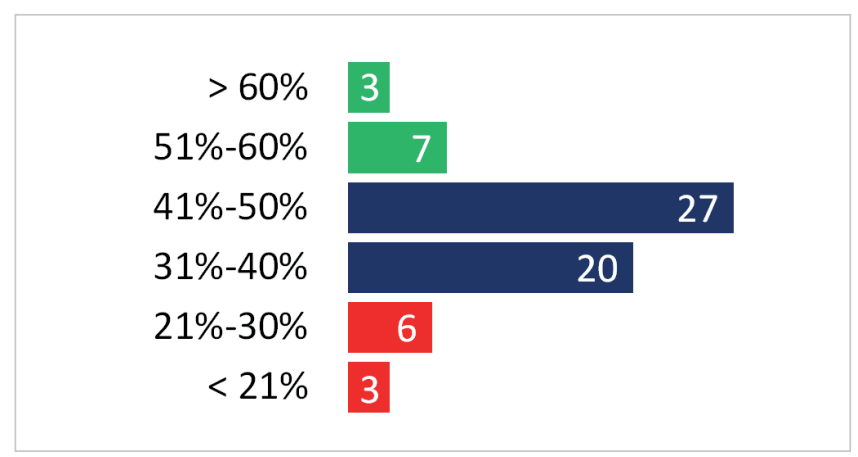

Gráfico 2. Distribución del número universidades españolas según su Tasa de cobertura en Altmetric.com de trabajos en Web of Science publicados en 2014-2016

WoS en estas áreas, así como Barcelona con el $77 \%$ o la $A u$ tònoma de Barcelona con el 58\%).

\subsection{Principales indicadores altmétricos de las univer- sidades}

Para determinar la importancia de los diferentes indicadores se ha realizado un análisis de componentes principales (PCA) (Gray, 2017) que señala la existencia de cuatro componentes que acumulan el 95\% de toda la varianza (tabla 4).

El primer componente principal explica hasta un $82 \%$ de la varianza y estaría conformado por cuatro de los indicadores: Twitter, noticias, Facebook y Wikipedia. Excepto Wikipedia, todas las plataformas presentan elevados grados de cobertura.

El resto de componentes explican poco la varianza; por ejemplo, el Componente 2 (integrado por Policy documents y F1000) sólo consigue cubrir el 5\%. El análisis confirma que para estudiar la visibilidad de las universidades, únicamente habría que tener en cuenta un número reducido de indicadores, que además coinciden con aquellos que presentan un mayor grado de cobertura.

La tabla 5 incluye las 10 primeras universidades españolas por menciones en las plataformas identificadas en el Componente 1. La Universitat de Barcelona es líder en mencio-

Tabla 3. Principales Tasas de cobertura en Altmetric.com (TCA) de los trabajos en Web of Science (2014-2016) publicados por las universidades españolas

\begin{tabular}{|c|c|c|c|c|c|}
\hline Universidades con mejor TCA & N. trabajos & $\begin{array}{l}\text { TCA } \\
(\%)\end{array}$ & $\begin{array}{l}\text { TCA para las universidades } \\
\text { más productivas }\end{array}$ & N. trabajos & TCA (\%) \\
\hline Universitat Pompeu Fabra & 3.272 & 71 & Universitat de Barcelona & 16.482 & 60 \\
\hline Universitat Internacional de Catalunya & 390 & 67 & Universitat Autònoma de Barcelona & 10.077 & 56 \\
\hline Universidad de Deusto & 406 & 66 & Universidad Complutense de Madrid & 7.923 & 42 \\
\hline Universitat de Barcelona & 16.482 & 60 & Universitat de València & 7.208 & 48 \\
\hline Universitat de Vic & 194 & 60 & Universidad de Granada & 6.704 & 42 \\
\hline Universidades con peor TCA & & & Universidad Autónoma de Madrid & 6.341 & 50 \\
\hline Universidad Pontificia de Salamanca & 18 & 11 & Universidad del País Vasco & 6.214 & 42 \\
\hline Universidad Antonio de Nebrija & 67 & 16 & Universidad de Sevilla & 5.150 & 39 \\
\hline Universidad Pontificia de Comillas & 227 & 19 & Universidad de Zaragoza & 4.660 & 39 \\
\hline Universidad Internacional de La Rioja & 137 & 25 & Universitat Politècnica de València & 4.414 & 48 \\
\hline Universidad Politécnica de Cartagena & 742 & 25 & & & \\
\hline
\end{tabular}

* TCA: Tasa de cobertura en Altmetric.com 
nes en las cuatro plataformas con porcentajes que oscilan entre el $25 \%$ y el $28 \%$ sobre el total de menciones del conjunto de las universidades. En general, las universidades con sede en Barcelona (Pompeu Fabra, Barcelona y Autònoma de Barcelona) se sitúan en todos los casos entre los cuatro primeros puestos. Las madrileñas Universidad Complutense y la Autónoma de Madrid conforman el otro núcleo de universidades ya que aparecen en las cuatro clasificaciones. Otras universidades de corte generalista, como son Granada, València, País Vasco y Oviedo también logran puestos relevantes. Entre las privadas sólo la Universidad de Navarra consigue aparecer entre las diez primeras en Twitter y Facebook.

Las universidades con mayor producción científica WoS son las que tienen un mayor número de menciones en los indicadores del Componente 1. Sin embargo la correlación entre el número de artículos WoS y menciones no es perfecta $(0,78)$, detectándose algunas anomalías. Por ejemplo
Tabla 4. Análisis de componentes principales para determinar el peso de las diferentes métricas incluidas en Altmetric. com analizadas para las universidades españolas

\begin{tabular}{|l|c|c|c|c|}
\hline & Componente 1 & Componente 2 & Componente 3 & Componente 4 \\
\hline Porcentaje de la varianza explicada & $82,23 \%$ & $5,68 \%$ & $4,10 \%$ & $3,01 \%$ \\
\hline Porcentaje acumulado de la varianza & $82,23 \%$ & $87,91 \%$ & $92,01 \%$ & $95,02 \%$ \\
\hline $\begin{array}{l}\text { Plataformas que influyen en los } \\
\text { componentes }\end{array}$ & $\begin{array}{l}\text { Twitter, } \\
\text { noticias, } \\
\text { Facebook, } \\
\text { Wikipedia }\end{array}$ & $\begin{array}{l}\text { Informes } \\
\text { técnicos, } \\
\text { F1000 }\end{array}$ & $\begin{array}{l}\text { Weibo, } \\
\text { Portales de } \\
\text { expertos }\end{array}$ & $\begin{array}{l}\text { Blogs, } \\
\text { Google+ } \\
\text { vídeos }\end{array}$ \\
\hline
\end{tabular}

la Universitat Pompeu Fabra y la de Barcelona obtienen un mayor número de menciones de las que les correspondería. La Universitat de Barcelona a nivel nacional acumula el 17\% de las citas de todo el sistema universitario, pero en el caso de las altmétricas acumula entre el $25 \%-28 \%$. Un caso similar ocurre con la Universidad de La Laguna que obtiene una alta puntuación en news stories y Wikipedia.

En el caso contrario, las universidades orientadas a la ingeniería y ciencias sociales, que ya vienen lastradas por su peor cobertura en Web of Science, no aparecen en posiciones relevantes pese a encontrarse algunas de ellas entre las más productivas (Politècnica de València, Politécnica de Madrid, Politècnica de Catalunya).

Tabla 5. Ranking de las diez primeras universidades según el número de menciones en diferentes plataformas (Twitter, news stories, Facebook y Wikipedia) incluidas en el Componente 1.

\begin{tabular}{|c|c|c|}
\hline \multicolumn{3}{|l|}{ Twitter } \\
\hline Universidad & $\begin{array}{c}\text { Número } \\
\text { menciones }\end{array}$ & $\begin{array}{l}\text { Porcentaje } \\
\text { sobre total }\end{array}$ \\
\hline Universitat de Barcelona & 88.601 & 26 \\
\hline Universitat Autònoma de Barcelona & 41.407 & 12 \\
\hline Universitat Pompeu Fabra & 40.652 & 12 \\
\hline Universitat de València & 31.990 & 9 \\
\hline Universidad Autónoma de Madrid & 24.208 & 7 \\
\hline Universidad Complutense de Madrid & 23.703 & 7 \\
\hline Universidad de Granada & 16.912 & 5 \\
\hline Universidad del País Vasco & 16.528 & 5 \\
\hline Universidad de Navarra & 15.004 & 4 \\
\hline Universidad de Oviedo & 14.354 & 4 \\
\hline \multicolumn{3}{|l|}{ Facebook } \\
\hline Universidad & $\begin{array}{c}\text { Número } \\
\text { menciones }\end{array}$ & $\begin{array}{l}\text { Porcentaje } \\
\text { sobre total }\end{array}$ \\
\hline Universitat de Barcelona & 7.754 & 28 \\
\hline Universitat Autònoma de Barcelona & 3.196 & 12 \\
\hline Universidad Complutense de Madrid & 2.681 & 10 \\
\hline Universitat Pompeu Fabra & 2.504 & 9 \\
\hline Universitat de València & 2.364 & 9 \\
\hline Universidad Autónoma de Madrid & 1.559 & 6 \\
\hline Universidad de Granada & 1.507 & 5 \\
\hline Universidad de Navarra & 1.280 & 5 \\
\hline Universidad del País Vasco & 1.266 & 5 \\
\hline Universidad de Oviedo & 1.065 & 4 \\
\hline
\end{tabular}

\begin{tabular}{|c|c|c|}
\hline \multicolumn{3}{|l|}{ News stories } \\
\hline Universidad & $\begin{array}{c}\text { Número } \\
\text { menciones }\end{array}$ & $\begin{array}{l}\text { Porcentaje } \\
\text { sobre total }\end{array}$ \\
\hline Universitat de Barcelona & 7.104 & 25 \\
\hline Universitat Autònoma de Barcelona & 3.632 & 13 \\
\hline Universitat Pompeu Fabra & 3.202 & 11 \\
\hline Universitat de València & 2.964 & 10 \\
\hline Universidad Autónoma de Madrid & 2.493 & 9 \\
\hline Universidad Complutense de Madrid & 2.202 & 8 \\
\hline Universidad del País Vasco & 2.112 & 7 \\
\hline Universidad de Oviedo & 1.489 & 5 \\
\hline Universidad de Granada & 1.471 & 5 \\
\hline Universidad de La Laguna & 1.406 & 5 \\
\hline \multicolumn{3}{|l|}{ Wikipedia } \\
\hline Universidad & $\begin{array}{c}\text { Número } \\
\text { menciones }\end{array}$ & $\begin{array}{l}\text { Porcentaje } \\
\text { sobre total }\end{array}$ \\
\hline Universitat de Barcelona & 510 & 26 \\
\hline Universitat Pompeu Fabra & 426 & 22 \\
\hline Universidad de Extremadura & 226 & 12 \\
\hline Universitat Autònoma de Barcelona & 178 & 9 \\
\hline Universidad Autónoma de Madrid & 158 & 8 \\
\hline Universidad Complutense de Madrid & 152 & 8 \\
\hline Universidad de La Laguna & 141 & 7 \\
\hline Universitat de València & 120 & 6 \\
\hline Universidad de Granada & 87 & 4 \\
\hline Universidad del País Vasco & 81 & 4 \\
\hline
\end{tabular}


Uno de los factores esenciales que está determinando la posición en los rankings es la capacidad de publicar trabajos con una gran difusión, como refleja su Altmetric attention score. Estos trabajos con gran impacto altmétrico se publican en las revistas multidisciplinares y biomédicas más prestigiosas (Nature, Science, PNAS, Lancet, New England, JAMA, entre otras). Esta situación indica que el número de menciones globales de las universidades está determinado por el factor disciplinar y por la publicación en revistas de alto impacto, que además gozan de gran difusión mediática. A modo ilustrativo en la tabla 6 se observa qué tipo de revistas son las que más altmétricas acaparan para las tres universidades más destacadas de este estudio.

\section{Discusión y conclusiones}

Este trabajo es el primero en recopilar y calcular las altmétricas de las universidades de un país en su conjunto. Se han estudiado los artículos recogidos en Web of Science para el período 2014-2016, de los cuales un 92\% disponen de DOI (115.838 documentos), una cifra similar al 93\% que Torres-Salinas, Robinson-García y Jiménez-Contreras (2016) proporcionan en su estudio sobre cuatro universidades españolas y al $90 \%$ reportado por Gorraiz et al. (2016) en un estudio global sobre Web of Science.

Cabe destacar que las universidades españolas no difieren demasiado del conjunto de Web of Science a tenor de estos datos. El porcentaje de trabajo con DOI es un dato importante al tratarse del identificador empleado para recuperar información de Altmetric.com.

Otro de los principales resultados corrobora cómo un reducido número de plataformas genera la gran mayoría de menciones. Si excluimos Mendeley, el $82 \%$ de menciones corresponde a Twitter, seguida de lejos por las noticias (7\%) y Facebook (5\%). Thelwall et al. (2013) indican que, con la excepción de Twitter, la cobertura de las altmétricas es baja: por debajo del $20 \%$ en todos los casos y en muchos casos sustancialmente más reducida. Esta información se refuerza a través del análisis de componentes principales, donde el componente 1 , compuesto por las 3 redes anteriores más Wikipedia, supone el $82 \%$ de la varianza explicada total.
Estos datos pueden ayudarnos a concentrar esfuerzos en aquellos indicadores que proporcionan información útil y relevante estadísticamente en lugar de en ampliar el número de fuentes.

La diversa naturaleza de las plataformas contempladas merece hacer una reflexión acerca del diverso grado de esfuerzo que implica la generación de una mención en cada una de ellas. El esfuerzo en la edición de un artículo de Wikipedia o en la redacción de una noticia es considerablemente mayor que en una publicación en Twitter, lo cual permite explicar la diferencia en el volumen de menciones entre unas fuentes y otras.

Se ha determinado asimismo que el $42 \%$ de los artículos WoS están indexados en Altmetric.com. Un valor muy superior al 19\% reportado por Robinson-García et al. (2014) en un estudio global de Altmetric.com para el período 2011-2013. Estas diferencias pueden indicar la mejora de la cobertura para los años más recientes. La cifra de cobertura muestra diferencias entre universidades con una elevada dispersión del porcentaje de artículos recogidos en Altmetric.com: desde el $71 \%$ de la Universitat Pompeu Fabra al $11 \%$ de la Pontificia de Salamanca. El desequilibrio se podría explicar por el perfil disciplinar ya que, como indican Torres-Salinas, Robinson-García y Jiménez-Contreras (2016), las tasas de cobertura son claramente más bajas en áreas como ingeniería, humanidades y ciencias sociales. Hammarfelt (2014) aborda el caso de las publicaciones en humanidades más centradas en formatos impresos y monografías, dando lugar a un peso menor en estos indicadores altmétricos.

En el caso español las humanidades y ciencias sociales tienen menor grado de internacionalización, lo que conlleva que gran parte de su producción se publique en revistas WoS nacionales, lo que podría limitar su difusión y potenciales menciones. Sí resulta evidente que los trabajos con mayor número de altmétricas corresponden a los publicados en revistas de alto impacto (por ejemplo, Science y Nature) y los publicados en las áreas de biomedicina y biología (tabla 6); una situación que también se produce a nivel global ya que por ejemplo de los 100 trabajos más divulgados en 2017 de acuerdo con Altmetric.com (2017b) 53 correspon-

Tabla 6. Revista donde han aparecido publicados los trabajos con mayor Altmetric Score para las tres universidades con mejores indicadores altmétricos

\begin{tabular}{|c|c|c|c|c|c|}
\hline \multicolumn{2}{|c|}{ Universitat de Barcelona } & \multicolumn{2}{|c|}{ Universitat Pompeu Fabra } & \multicolumn{2}{|c|}{ Universitat Autònoma de Barcelona } \\
\hline $\begin{array}{c}\text { Altmetric } \\
\text { score }\end{array}$ & Revista & $\begin{array}{c}\text { Altmetric } \\
\text { score }\end{array}$ & Revista & $\begin{array}{c}\text { Altmetric } \\
\text { score }\end{array}$ & Revista \\
\hline 2.519 & PLoS one & 1.740 & Lancet & 1.390 & Lancet diabetes \\
\hline 1.740 & Lancet & 1.281 & Lancet & 1.126 & Nature genetics \\
\hline 1.390 & Lancet diabetes & 1.247 & Nature & 974 & Scientific reports \\
\hline 1.281 & Lancet & 1.060 & Preventive medicine & 966 & Science \\
\hline 1.169 & Lancet & 933 & Nature & 918 & JAMA internal medicine \\
\hline 1.094 & JAMA internal medicine & 746 & Nature & 800 & $J A M A$ \\
\hline 1.068 & Lancet & 698 & Lancet haematology & 665 & Scientific reports \\
\hline 922 & PNAS & 685 & Nature & 588 & Physical review letters \\
\hline 918 & JAMA internal medicine & 679 & PNAS & 578 & Biology \\
\hline 893 & Physical review letters & 646 & Nature genetics & 522 & Science \\
\hline
\end{tabular}

Nota: El listado de los trabajos con mayor visibilidad para cada universidad se puede consultar en el ranking Knowmetrics en "Top papers universidades": http://altmetrics.knowmetrics.org/ranking/univ2 
dían al área de medicina y 20 a biología. En el caso de las universidades españolas (Torres-Salinas, 2017) se observa como, empleando la misma base de datos usada en este trabajo, 523 artículos (1\%) son capaces de acaparar el 50\% de las menciones en buena parte de las plataformas. Por tanto, las universidades con mayor producción en revistas de alto impacto y en disciplinas biomédicas estarán en disposición de obtener un mayor número de menciones.

\section{Las universidades con mayor producción en revistas de alto impacto y en discipli- nas biomédicas estarán en disposición de obtener un mayor número de menciones}

Finalmente, los resultados expuestos y la difusión pública de los datos a través del ranking Knowmetrics deberían ayudar a reflexionar sobre las estrategias digitales de las universidades. El fomento de una cultura digital entre los investigadores, el diseño de planes de difusión de la investigación integrales y coordinados, las buenas prácticas en la referenciación de publicaciones en las redes, la elección de las plataformas adecuadas son sólo algunos de los aspectos que deberían considerarse si realmente se quiere optimizar la comunicación social de resultados y la visibilidad global institucional en la Web y sus múltiples ecosistemas.

\section{Agradecimientos}

Este trabajo ha sido posible gracias a la financiación del proyecto "Knowmetrics: evaluación del conocimiento en la sociedad digital" dentro de las ayudas de la Fundación BBVA a equipos de investigación científica 2016.

Agradecemos a Altmetric.com la cesión de los datos que han permitido la realización de este trabajo.

\section{Referencias}

Altmetric.com (2017a). "How is the Altmetric Attention Score calculated?". Altmetric support.

https://goo.gl/4hvTPp

Altmetric.com (2017b). "Top 100 articles 2017". Altmetric.com. https://www.altmetric.com/top100/2017/\#list

Bar-llan, Judit (2008). "Informetrics at the beginning of the $21^{\text {st }}$ century - A review". Journal of informetrics, v. 2, n. 1, pp. 1-52. https://goo.gl/s72eqr https://doi.org/10.1016/j.joi.2007.11.001

Bornmann, Lutz (2014). “Do altmetrics point to the broader impact of research? An overview of benefits and disadvantages of altmetrics". Journal of informetrics, v. 8, n. 4, pp. 895-903.

https://arxiv.org/abs/1406.7091

https://doi.org/10.1016/j.joi.2014.09.005

Costas, Rodrigo; Zahedi, Zohreh; Wouters, Paul (2015). “Do 'altmetrics' correlate with citations? Extensive comparison of altmetric indicators with citations from a multidisciplinary perspective". Journal of the Association for Information Science and Technology, v. 66, n. 10, pp. 2003-2019. https://openaccess.leidenuniv.nl/handle/1887/23041 https://doi.org/10.1002/asi.23309

Gorraiz, Juan; Melero-Fuentes, David; Gumpenberger, Christian; Valderrama-Zurián, Juan-Carlos (2016). "Availability of digital object identifiers (DOIs) in Web of Science and Scopus". Journal of informetrics, v. 10, n. 1, pp. 98-109. https://goo.gl/JkR47f https://doi.org/10.1016/j.joi.2015.11.008

Gray, Virginia (2017). Principal component analysis: Methods, applications \& technology. Nova Science Publishers Inc., Mathematics Research Development. ISBN: 978 1536108897

Gumpenberger, Christian; Glänzel, Wolfgang; Gorraiz, Juan (2016). "The ecstasy and the agony of the Altmetric score". Scientometrics, v. 108, n. 2, pp. 977-982.

https://doi.org/10.1007/s11192-016-1991-5

Hammarfelt, Björn (2014). "Using altmetrics for assessing research impact in the humanities". Scientometrics, v. 101, n. 2, pp. 1419-1430.

https://doi.org/10.1007/s11192-014-1261-3

Haustein, Stefanie (2016). "Grand challenges in altmetrics: Heterogeneity, data quality and dependencies". Scientometrics, v. 108, n. 1, pp. 413-423.

https://arxiv.org/abs/1603.04939

https://dx.doi.org/10.1007/s11192-016-1910-9

Haustein, Stefanie; Bowman, Timothy D.; Costas, Rodrigo (2015). "Interpreting 'altmetrics': viewing acts on social media through the lens of citation and social theories". In: Sugimoto, Cassidy R. (ed.). Theories of informetrics: A festschrift in honor of Blaise Cronin.

http://arxiv.org/abs/1502.05701

Haustein, Stefanie; Peters, Isabella; Bar-Ilan, Judit; Priem, Jason; Shema, Hadas; Terliesner, Jens (2014a). "Coverage and adoption of altmetrics sources in the bibliometric community". Scientometrics, v. 101, n. 2, pp. 1145-1163. https://doi.org/10.1007/s11192-013-1221-3

Haustein, Stefanie; Peters, Isabella; Sugimoto, Cassidy R.; Thelwall, Mike; Larivière, Vincent (2014b). "Tweeting biomedicine: An analysis of tweets and citations in the biomedical literature". Journal of the Association for Information Science and Technology, v. 65, n. 4, pp. 656-669.

https://arxiv.org/abs/1308.1838

https://doi.org/10.1002/asi.23101

Hicks, Diana; Wouters, Paul; Waltman, Ludo; De-Rijcke, Sarah; Ràfols, Ismael (2015). "The Leiden Manifesto for research metrics". Nature, v. 520, n. 7548, pp. 429-431 https://www.nature.com/news/bibliometrics-the-leidenmanifesto-for-research-metrics-1.17351

Liu, Jean; Adie, Euan (2013). "Five challenges in altmetrics: A toolmaker's perspective". Bulletin of the Association for Information Science and Technology, v. 39, n. 4, pp. 31-34. https://goo.gl/6P1nY8 https://doi.org/10.1002/bult.2013.1720390410

Martín-Martín, Alberto; Orduña-Malea, Enrique; Delgado-López-Cózar, Emilio (2016). "The role of ego in academic profile services: Comparing Google Scholar, ResearchGate, 
Mendeley, and ResearcherID".

https://doi.org/10.2139/ssrn.2745892

Mohammadi, Ehsan; Thelwall, Mike (2014). "Mendeley readership altmetrics for the social sciences and humanities: Research evaluation and knowledge flows". Journal of the Association for Information Science and Technology, v. 65, n. 8, pp. 1627-1638.

https://goo.gl/Yj3gD7

https://doi.org/10.1002/asi.23071

Peters, Isabella; Kraker, Peter; Lex, Elisabeth; Gumpenberger, Christian; Gorraiz, Juan (2016). "Research data explored: An extended analysis of citations and altmetrics". Scientometrics, v. 107, n. 2, pp. 723-744.

https://doi.org/10.1007/s11192-016-1887-4

Piwowar, Heather (2013a). "Altmetrics: Value all research products". Nature, v. 493, n. 7431, pp. 159-159.

https://doi.org/10.1038/493159a

Piwowar, Heather (2013b). "Introduction altmetrics: what, why and where?". Bulletin of the Association for Information Science and Technology, v. 39, n. 4, pp. 8-9.

https://doi.org/10.1002/bult.2013.1720390404

Priem, Jason; Hemminger, Bradley M. (2010). "Scientometrics 2.0: Toward new metrics of scholarly impact on the social web. First Monday, v. 15, n. 7.

http://journals.uic.edu/ojs/index.php/fm/article/view/2874/2570

Priem, Jason; Piwowar, Heather A.; Hemminger, Bradley M. (2012). "Altmetrics in the wild: Using social media to explore scholarly impact".

http://arxiv.org/html/1203.4745

Priem, Jason; Taraborelli, Dario; Groth, Paul; Neylon, Cameron (2010). "Altmetrics: A Manifesto". Altmetrics.org. http://altmetrics.org/manifesto

Robinson-García, Nicolás; Torres-Salinas, Daniel; Zahedi, Zohreh; Costas, Rodrigo (2014). "New data, new possibilities: Exploring the insides of Altmetric.com". El profesional de la information, v. 23, n. 4, pp. 359-366.

https://doi.org/10.3145/epi.2014.jul.03

Romero-Frías, Esteban; Vaughan, Liwen (2012). “Exploring the relationships between media and political parties through web hyperlink analysis: The case of Spain". Journal of the American Society for Information Science and Technology, v. 63, n. 5, pp. 967-976.

https://doi.org/10.1002/asi.22625

Sud, Pardeep; Thelwall, Mike (2014). "Evaluating altmetrics". Scientometrics, v. 98, n. 2, pp. 1131-1143.

https://goo.gl/vwnygr

https://doi.org/10.1007/s11192-013-1117-2

Thelwall, Mike (2008). "Bibliometrics to webometrics". Journal of information science, v. 34, n. 4, pp. 605-621. https://goo.gl/S6GaEo https://doi.org/10.1177/0165551507087238

Thelwall, Mike; Haustein, Stefanie; Larivière, Vincent; Sugimoto, Cassidy R. (2013). "Do altmetrics work? Twitter and ten other social web services". PloS one, v. 8, n. 5, pp. e64841. https://doi.org/10.1371/journal.pone.0064841

Thelwall, Mike; Vaughan, Liwen; Björneborn, Lennart (2005). "Webometrics". Annual review of information science and technology, v. 39, n. 1, pp. 81-135.

https://doi.org/10.1002/aris.1440390110

Torres-Salinas, Daniel (2017). “Altmetric beauties. ¿Cuáles son los trabajos científicos con mayor impacto en las redes sociales?". En: I Congreso internacional "Territorios digitales". Universidad de Granada, Medialab UGR, Granada, 29-30 junio 2017. http://hdl.handle.net/10481/47115

Torres-Salinas, Daniel; Cabezas-Clavijo, Álvaro; Jiménez-Contreras, Evaristo (2013). "Altmetrics: nuevos indicadores para la comunicación científica en la Web 2.0". Comunicar, v. 21, n. 41.

https://doi.org/10.3916/C41-2013-05

Torres-Salinas, Daniel; Gumpenberger, Christian; Gorraiz, Juan (2017). "PlumX as a potential tool to assess the macroscopic multidimensional impact of books". Frontiers in research metrics and analytics, v. 2, n. 5.

https://doi.org/10.3389/frma.2017.00005

Torres-Salinas, Daniel; Milanés-Guisado, Yusnelkis (2014). "Presencia en redes sociales y altmétricas de los principales autores de la revista 'El profesional de la información'”. El profesional de la información, v. 23, n. 4, pp. 367-372.

https://doi.org/10.3145/epi.2014.jul.04

Torres-Salinas, Daniel; Robinson-García, Nicolás; Gorraiz, Juan (2017). "Filling the citation gap: Measuring the multidimensional impact of the academic book at institutional level with PlumX". Scientometrics, v. 113, n. 3, pp. 1371-1384. https://arxiv.org/abs/1710.00368 https://doi.org/10.1007/s11192-017-2539-z

Torres-Salinas, Daniel; Robinson-García, Nicolás; Jiménez-Contreras, Evaristo (2016). "Can we use altmetrics at the institutional level? A case study analysing the coverage by research areas of four Spanish universities". https://arxiv.org/abs/1606.00232

Vaughan, Liwen; Wu, Guozhu (2004). "Links to commercial websites as a source of business information". Scientometrics, v. 60, n. 3, pp. 487-496.

https://doi.org/10.1023/B:SCIE.0000034389.14825.bc

Weller, Martin (2011). The digital scholar. How technology is transforming scholarly practice. Bloomsbury. ISBN: 9781 849664974

Wilsdon, James; Allen, Liz; Belfiore, Eleanora; Campbell, Philip; Curry, Stephen; Hill, Steven; Jones, Richard; Kain, Roger; Kerridge Simon; Thelwall, Mike; Tinkler, Jane; Viney, Ian; Wouters, Paul; Hill, Jude; Johnson, Ben (2015). The metric tide: Report of the independent review of the role of metrics in research assessment and management.

https://dx.doi.org/10.13140/RG.2.1.4929.1363

Wouters, Paul; Costas, Rodrigo (2012). Users, narcissism and control - tracking the impact of scholarly publications in the $21^{\text {st }}$ century. Utrecht: SURF-foundation.

http://research-acumen.eu/wp-content/uploads/Usersnarcissism-and-control.pdf 